Bio - grafía. Escritos sobre la Biología y su Enseñanza. ISSN 2027-1034

Edición Extraordinaria. p.p. 1314 - 1324

Memorias del IX Encuentro Nacional de Experiencias en Enseñanza de la Biología y la Educación Ambiental. IV Congreso Nacional de Investigación en Enseñanza de la Biología.

\title{
EL DESARROLLO DE LA OBSERVACIÓN Y LA INDAG ACIÓN EN ESTUDIANTES DE GRADO DÉCIMO DE LA IE ÉRIKA BEATRÍZ DE MAICAO - LA GUAJIRA
}

\author{
Francisca Toscano ${ }^{1}$ \\ Carlos Humberto Barreto Tovar ${ }^{2}$
}

\section{RESUMEN}

La investigación se realizó en estudiantes del grado décimo de la institución educativa № 13 sede Erika Beatriz, en el municipio de Maicao la Guajira, los cuales presentan bajos niveles de indagación y observación; para esto se desarrollaron planeaciones de clase desde la Enseñanza para la compresión (EpC). Con el propósito de identificar los niveles de observación e indagación de los educandos, se realizaron salidas por las dependencias del colegio, posteriormente los estudiantes elaboraron un escrito con relación a todo lo observado. De igual forma se plantearon preguntas relacionadas con sus observaciones. Desde el marco de la EpC se plantearon actividades que permitieran tener una compresión de la manera cómo están algunas habilidades científicas; específicamente, la indagación y la observación.

Para la obtención de resultados se organizó la información en matrices y gráficas, teniendo como referencia Niveles del proceso de observación según Santelices L. (1989) y formulación de preguntas (Furman \& García, 2014), logrando como resultado, que los estudiantes del grado décimo, demuestran bajos niveles de observación e indagación; de igual forma el interés de los maestros por cambiar las prácticas pedagógicas y enriquecerlas. Cabe destacar, que el docente como ente responsable del proceso de aprendizaje de los educandos debe implementar estrategias innovadoras para enseñar y aprender a investigar, de esta manera, también desarrollar las competencias de observación e indagación.

PALABRAS CLAVES: Observación, indagación, competencias científicas, desarrollo del pensamiento científico, Enseñanza para la Comprensión.

\section{ABSTRACT}

The research was carried out in students of the tenth grade of educational institution $\mathrm{N}^{\circ}$ 13 Erika Beatriz, in the municipality of Maicao la Guajira, which have low levels of inquiry and observation; for this class planning was developed from the Teaching for

\footnotetext{
${ }^{1}$ Universidad de La Sabana. Facultad de Educación. Maestría en Pedagogía Ext. La Guajira. Correo: franciscato@unisabana.edu.co

${ }_{2}$ Universidad de La Sabana. Facultad de Educación. Maestría en Pedagogía Ext. La Guajira. Correo: carlos.barreto2@unisabana.edu.co
} 


\title{
Bio - grafía. Escritos sobre la Biología y su Enseñanza. ISSN 2027-1034
}

\section{Edición Extraordinaria. p.p. 1314 - 1324}

\author{
Memorias del IX Encuentro Nacional de Experiencias en Enseñanza de la Biología y la \\ Educación Ambiental. IV Congreso Nacional de Investigación en Enseñanza de la \\ Biología.
}

Compression $(\mathrm{EpC})$. In order to identify the levels of observation and inquiry of students, departures were made by the school's departments, later students wrote a letter in relation to everything observed. Likewise, questions related to his observations were raised. From the framework of the $\mathrm{EpC}$, activities were proposed that allowed a compression of the way some scientific abilities are; specifically, inquiry and observation.

In order to obtain results, information was organized in matrices and graphs, having as reference Levels of the observation process according to Santelices L. (1989) and formulation of questions (Furman \& García, 2014), resulting in the students of the degree tenth, show low levels of observation and inquiry; as well as teachers' interest in changing teaching practices and enriching them. It should be noted that teachers as responsible for the learning process of learners must implement innovative strategies to teach and learn to research, in this way also develop the skills of observation and inquiry.

KEY WORDS: Observation, inquiry, scientific competences, development of scientific thinking, Teaching for Understanding

\section{INTRODUCCIÓN}

La ciencia es tan común que a diario necesitamos de ella en diferentes contextos. Uno de ellos es la escuela. La enseñanza de las ciencias, especialmente de la biología y la educación ambiental, se ha convertido en algo que no es atrayente para nuestros alumnos, ya que estos la relacionan exclusivamente con los libros y consideran también, que sólo les compete a los científicos.

Son pocos los que se interesan por conocer más de la biología y la educación ambiental, debido a un sin número de problemas de índole social, económico, cultural; en las cuales se encuentran inmersos. Algunos estudiantes al ver las necesidades que se presentan en sus casas, se ven obligados a tener que conseguir dinero. Expresan que es mejor salir a trabajar que estar en las escuelas aprendiendo algo que no les servirá para la vida. Es allí donde la biología y la educación ambiental se evidencian como poco productivas.

Acevedo (1999) describe una situación muy común en el que hacer pedagógico cuando algunos estudiantes preguntan con mucha sinceridad para qué me sirve aprender un determinado tema, y en ocasiones se le da los argumentos posibles para que reflexione y la importancia de aprender ciencia. Pero ¿por qué surgen este tipo de preguntas en nuestros salones? Según Rurió, Vilches, Guisasa \& Romo (2001) afirman que: "todavía en las escuelas se sigue enseñando ciencia en forma conceptual y esto está arraigado en los docentes".

Es así como en la labor educativa, el docente debe implementar métodos que le permitan saber educar desde las condiciones históricas, sociales, económicas y políticas partiendo de sus reflexiones para promover la transformación de un saber. Preguntas como: De qué manera lo enseño, a quién enseño, dónde enseño, para qué; son interrogantes que permiten al docente tomar su conocimiento científico y transformarlo en un conocimiento 


\title{
Bio - grafía. Escritos sobre la Biología y su Enseñanza. ISSN 2027-1034
}

\section{Edición Extraordinaria. p.p. 1314 - 1324}

\author{
Memorias del IX Encuentro Nacional de Experiencias en Enseñanza de la Biología y la \\ Educación Ambiental. IV Congreso Nacional de Investigación en Enseñanza de la \\ Biología.
}

enseñado donde el estudiante aprende. De esta forma, el docente constantemente está trabajando en sus teorías pedagógicas para que los individuos puedan desempeñarse de manera productiva en la sociedad y a su vez desarrollar las competencias científicas.

En ese sentido, es importante desarrollar la competencia de la indagación y la observación en los alumnos, ya que es un proceso que se da en el pensamiento humano desde las primeras etapas de su desarrollo. También la indagación puede ser entendida como la habilidad para hacer preguntas, habilidad que tiene su origen en las necesidades del ser humano, el cual se convierte en un medio o instrumento para comprender y aprehender el objeto de estudio. Dewey (1929), señalaba que la pregunta y la curiosidad, en cuanto actitud exploratoria, es la que da origen al pensamiento, decía que en el niño la curiosidad es como un instinto natural y que en su crecimiento y participación en las relaciones sociales este se vale del lenguaje interrogativo (formulación de preguntas) para continuar explorando por medio de los adultos, el mundo.

\section{Contexto}

La Institución Educativa Erika Beatriz se encuentra ubicada en el municipio de Maicao en el departamento de la Guajira. La mayoría de los estudiantes son habitantes de los barrios aledaños al plantel y zona rural como la ranchería Chichitúi de donde algunos estudiantes deben recorrer distancias entre 15 y 30 minutos de caminata para llegar al colegio. La institución cuenta con una población de 1500 estudiantes desde los niveles de preescolar hasta undécimo grado.

Esta población se caracteriza por ser de escasos recursos, estrato socio económico bajo, con variadas actividades económicas ejercidas por los padres de familia. Entre los que se cuentan comerciantes, los cuales realizan intercambio comercial con Venezuela, otros son empacadores en la zona céntrica comercial de la ciudad, estos ganan dependiendo la cantidad de electrodomésticos que empaquen al día. Otros se desempeñan como albañiles y moto taxistas. En cuanto a las madres algunas son cabeza de familia y laboran como empleadas domésticas, dejando a los niños al cuidado de los hermanos mayores.

Por la posición geográfica, Maicao tiene una población multicultural y multiétnica, contando con estudiantes de la etnia Wayuu, zenues, afro descendientes, de diferentes partes de Colombia y Venezuela; los cuales llegan en forma repentina y luego de un tiempo desertan.

\section{Problemática}

El procesos de indagación empieza en las etapas tempranas del desarrollo humano, las cuales como padres, madres o docentes no se aprovechan para ir cultivando y desarrollando las competencias científicas, estas competencias son casi innatas y se van perdiendo a medidas que van creciendo, son esos jóvenes que ya no se asombran de lo que sucede, es normal todo lo que ocurre sin dedicarse a cuestionarse de su propia realidad. En esa línea se encontró bajo nivel de indagación en estudiantes de grado décimo, las preguntas que realizan se quedan meramente en conceptos, son superficiales, ya que no están familiarizados con el proceso y poco se trabaja en la escuela, el desarrollo de la habilidad para observar e indagar. 


\title{
Bio - grafía. Escritos sobre la Biología y su Enseñanza. ISSN 2027-1034
}

\section{Edición Extraordinaria. p.p. 1314 - 1324}

\author{
Memorias del IX Encuentro Nacional de Experiencias en Enseñanza de la Biología y la \\ Educación Ambiental. IV Congreso Nacional de Investigación en Enseñanza de la \\ Biología.
}

\section{METODOLOGÍA}

Enfoque cualitativo, descriptivo, Investigación Acción.

En la investigación se utilizó un enfoque cualitativo, ya que permite comprender el fenómeno que se analiza, en este caso se investiga la dimensión de aprendizaje con su categorías como la observación y la indagación, las cuales fueron agrupadas de tal manera que permitieran entender los diferentes niveles de indagación y observación en los que se encuentran los estudiantes. En cuanto al diseño se utilizó la investigación acción, esta permite al docente realizar reflexiones de su práctica pedagógica, para esto es necesaria la búsqueda de información teórica que le permita perfeccionar su quehacer para poder comprender su práctica y las diferentes situaciones que se presentan en el salón de clase. Para Stenhouse (1920) expresa que el docente es el medio más valioso de la escuela, este es una persona como cualquier otra, solo hay que proporcionarle oportunidad de perfeccionamiento y en particular para que afine su capacidad de juicio.

En los estudiantes del grado décimo, las edades oscilan entre los 15 y 17 años, son jóvenes pocos participativos, con frecuencia se ausentan del colegio sin causa justificada, en el desarrollo de actividades realizadas en el salón, se les pide que formulen preguntas; lo cual se les dificulta, demostrando no ser capaces de construir los interrogantes que le surgen. Teniendo en cuenta lo anterior fue necesario diseñar algunas actividades que permitieran identificar los niveles de observación e indagación. Para esto se realizó la planeación de clase por EpC. Se le pidió a los estudiantes realizar una salida de observación por el colegio, posteriormente, en un nuevo encuentro se le solicitó elegir una planta del jardín y realizar anotaciones en su cuaderno. De la misma manera con sus apuntes se les indicó que realizaran un escrito en forma de trabajo donde quedara plasmado todo lo observado en las dos salidas.

Con el documento realizado por los estudiantes se les pidió construir preguntas relacionadas con su escrito y las salidas realizadas, todo esto se recolectó y se realizó el respectivo análisis tabulando la información.

\section{RESULTADOS}

Se trabajó con los estudiantes de grado décimo, actividades para determinar los niveles de observación e indagación. Para la observación se tuvo como referente los niveles del proceso de observar según Santelices L. (1989).

Teniendo en cuenta lo anterior, se planearon sesiones de clase teniendo como referencia la planeación por Enseñanza para la Comprensión (EpC) en el que el hilo conductor se expresó de la siguiente manera: "Mi entorno hace parte de mí y me beneficia como persona"; el tópico generativo: "observo todo lo que está a mi alrededor"; a lo cual se trazaron unas metas de comprensión entre las que estaban: desarrollar la comprensión sobre el reconocimiento de los diferentes lugares del colegio como: salones, jardines, baños, etc., analizando en qué condiciones se encontraban con respecto a la utilidad que 
Bio - grafía. Escritos sobre la Biología y su Enseñanza. ISSN 2027-1034

Edición Extraordinaria. p.p. 1314 - 1324

Memorias del IX Encuentro Nacional de Experiencias en Enseñanza de la Biología y la

Educación Ambiental. IV Congreso Nacional de Investigación en Enseñanza de la Biología.

le dan todos los estudiantes. Cada meta comprensión como la conceptual, procedimental, actitudinal y de comunicación se plantearon bajo las siguientes preguntas: ¿por qué debemos cuidar lo público?, ¿cómo se recopila información mediante la observación y la indagación de diferentes aspectos relacionados con los bienes y recursos?, ¿por qué es importantes el cuidado de los bienes y recursos de su entorno escolar?, ¿cómo se puede organizar toda la información recolectada sobre la observación por el colegio para darla a conocer a tanto a mis compañeros y profesor?

Luego de sistematizar la información se obtienen los siguientes resultados:

\section{Gráfica 1}

Tabulación de los resultados niveles de observación

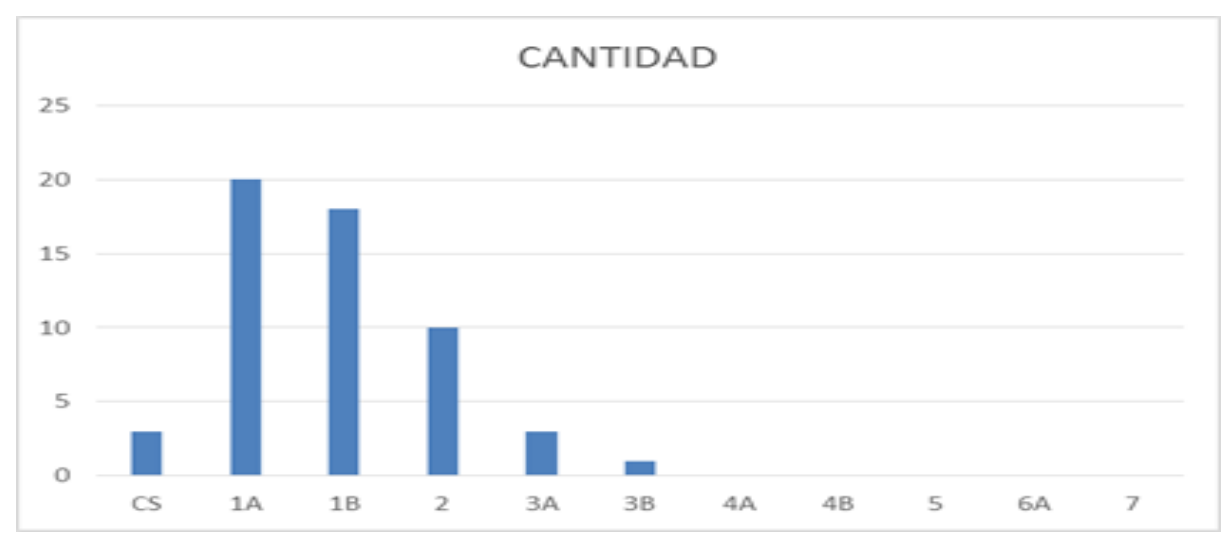

\section{Grafica 2.}

Porcentaje de estudiantes niveles de observación

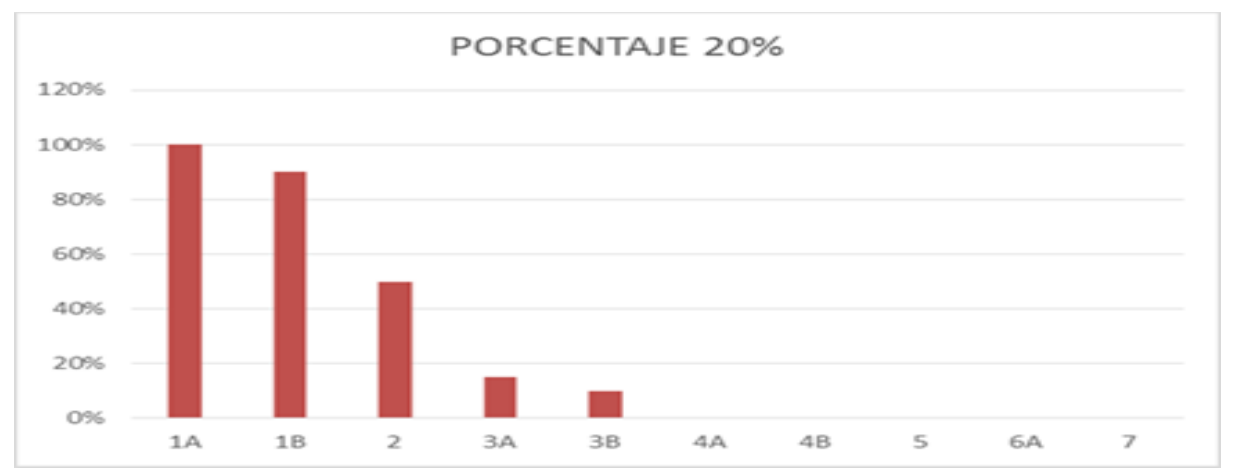


Bio - grafía. Escritos sobre la Biología y su Enseñanza. ISSN 2027-1034

Edición Extraordinaria. p.p. 1314 - 1324

Memorias del IX Encuentro Nacional de Experiencias en Enseñanza de la Biología y la Educación Ambiental. IV Congreso Nacional de Investigación en Enseñanza de la Biología.

\section{Gráfica 3}

\section{Tipología de las preguntas}

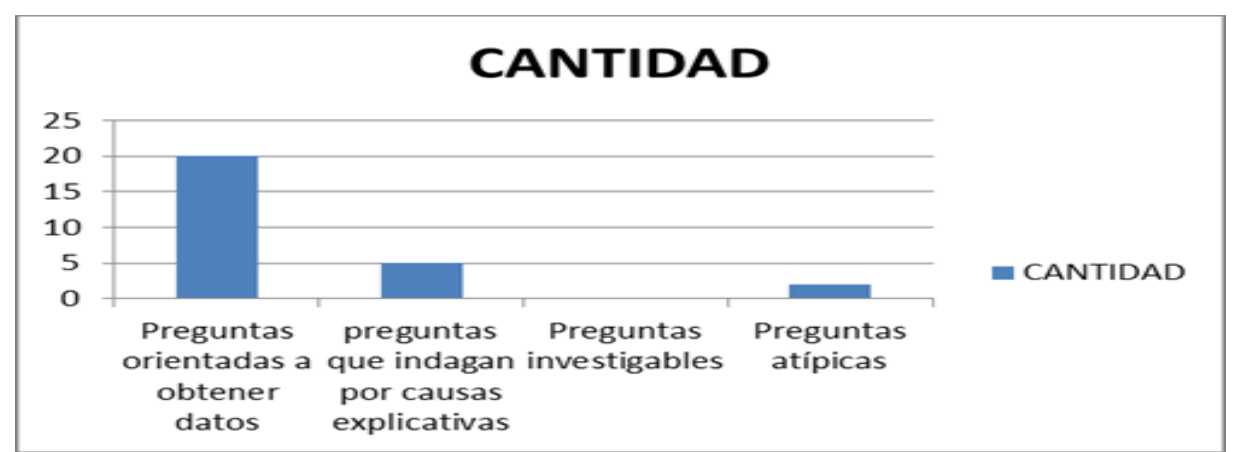

\section{Gráfica 4.}

\section{Porcentaje de tipología de las preguntas}

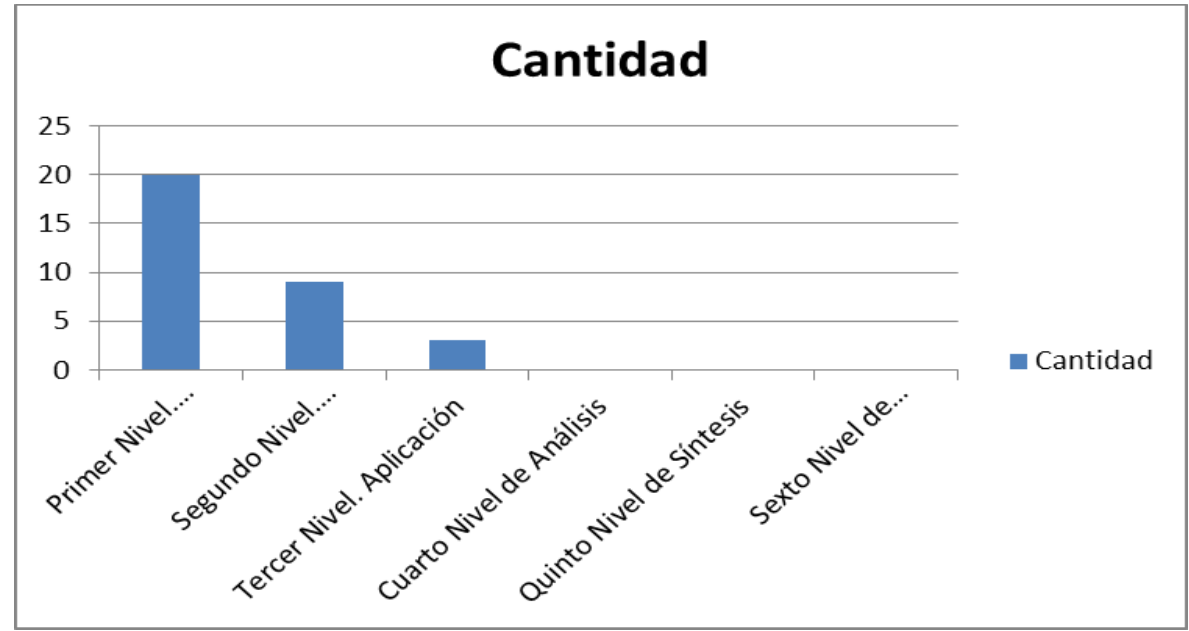

Teniendo en cuenta las habilidades de pensamiento científico, la observación según Santelices (1989) afirma que el observar para el niño como proceso científico es equivalente a las acciones que el hombre de ciencias realiza para desentrañar los misterios que el mundo encierra, propone que para observar se deben utilizar todos los sentidos.

En el análisis de las actividades se pudo comprobar que los estudiantes solo utilizan la visión y el olfato. En la primera actividad, salida por las dependencias del colegio para los estudiantes solo fue significativo la observación de los baños de las niñas a lo cual llegaron a la conclusión que están más deteriorados que el de los niños, encontrando que 
Bio - grafía. Escritos sobre la Biología y su Enseñanza. ISSN 2027-1034

Edición Extraordinaria. p.p. 1314 - 1324

Memorias del IX Encuentro Nacional de Experiencias en Enseñanza de la Biología y la Educación Ambiental. IV Congreso Nacional de Investigación en Enseñanza de la Biología.

no hay una sola marca en las paredes de estos, y que los olores que se desprenden de estos lugares son nauseabundos.

La mayor cantidad de preguntas realizadas por nuestros estudiantes se encuentran en el nivel 1 de observación, indicando que solo observan aspectos meramente superficiales como colores, olores. (Gráfica 1)

También se realizó una sesión de clase donde se proponía un hilo conductor el cual se planteó de la siguiente manera "Mis plantas reflejan lo que son internamente en sus genes? Y su tópico generativo ¿las plantas cuenta su hermosura al mirarlas? donde los estudiantes debían elegir una planta del jardín del colegio y realizar procesos de observación y sacar la mayor información de la planta. Luego estos debían presentar un escrito de las actividades propuestas. Estas actividades permitieron determinar en qué niveles se encontraban nuestros estudiantes en la observación y la indagación.

A continuación se muestra la siguiente tabla donde se evidencian algunas preguntas planteadas por los estudiantes y su clasificación.

\section{Tabla 1}

\section{Niveles del proceso de observación}

Niveles del proceso de observar según Santelices L.(1989) (Metodología de Ciencias Naturales para la enseñanza básica).

Nivel 1. ¿Por qué los baños tienen mal olor?

A. Identificar y denominar formas básicas y colores en objetos diversos.

B. describir, en términos elementales, sonidos y olores; describir y comparar tamaños, pesos, consistencia, dureza.

Nivel 2.

Describir objetos y seres a través de un conjunto de observaciones utilizando varios sentidos $e$ identificar en un conjunto de objetos y seres a uno de ellos dada su descripción.

Nivel 3:

A. formular observaciones cuantitativas acerca de los objetos y seres.

¿Por qué los estudiantes han cambiado los 
Bio - grafía. Escritos sobre la Biología y su Enseñanza. ISSN 2027-1034

Edición Extraordinaria. p.p. 1314 - 1324

Memorias del IX Encuentro Nacional de Experiencias en Enseñanza de la Biología y la Educación Ambiental. IV Congreso Nacional de Investigación en Enseñanza de la Biología.

\begin{tabular}{|l|l|l|}
\hline $\begin{array}{l}\text { B. Describir cambios producidos en } \\
\text { objetos y seres. }\end{array}$ & $\begin{array}{l}\text { cuadernos por las paredes de los baños? } \\
\text { ¿Qué pasaría si las plantas desaparecen } \\
\text { del ecosistema? }\end{array}$ \\
\hline $\begin{array}{l}\text { Nivel } 4 . \\
\begin{array}{l}\text { A. distinguir entre observaciones e } \\
\text { interpretaciones. }\end{array}\end{array}$ & No aplican \\
$\begin{array}{l}\text { B. formular sus descripciones evitando } \\
\text { introducir afirmaciones que no } \\
\text { constituyen observaciones. quar. }\end{array}$ & \\
\hline
\end{tabular}

En la tabla se clasificaron los diferentes tipos de preguntas que surgieron de la actividad de observación para Furman \& García (2014), adaptación de lo propuesto por Roca, Márquez \& Sanmartí (2013); De la misma manera se utilizó la propuesta de Maloka, (2003) "Cómo caracterizar los tipos de preguntas"

\begin{tabular}{|l|l|l|}
\hline Nivel & Categoría & Descripción \\
\hline Primer nivel & Conocimiento & $\begin{array}{l}\text { Recordar material aprendido con } \\
\text { anterioridad como hechos, términos, } \\
\text { conceptos básicos y respuestas. }\end{array}$ \\
\hline Segundo nivel & Comprensión & $\begin{array}{l}\text { Demostrar el entendimiento de hechos e } \\
\text { ideas organizando comparando, } \\
\text { traduciendo, interpretando, haciendo } \\
\text { descripciones y exponiendo las ideas } \\
\text { principales. }\end{array}$ \\
\hline Tercer nivel & Aplicación & $\begin{array}{l}\text { Resolver o solucionar problemas } \\
\text { aplicando el conocimiento adquirido, } \\
\text { hechos, técnicas y reglas, de manera } \\
\text { diferente. }\end{array}$ \\
\hline
\end{tabular}

En el análisis de los resultados se observa que la mayoría de los estudiantes del grado décimo se encuentra en nivel 1 en la tipología de las preguntas.

Para Márquez y Roca, (2006) "Las preguntas, han sido y son unos de los principales desencadenantes de las aportaciones científicas relevantes". Se afirma de la misma manera que las preguntas son fundamentales en el desarrollo científico, también lo son en el proceso de enseñanza y aprendizaje de las ciencias. 


\title{
Bio - grafía. Escritos sobre la Biología y su Enseñanza. ISSN 2027-1034
}

\section{Edición Extraordinaria. p.p. 1314 - 1324}

\author{
Memorias del IX Encuentro Nacional de Experiencias en Enseñanza de la Biología y la \\ Educación Ambiental. IV Congreso Nacional de Investigación en Enseñanza de la \\ Biología.
}

No se puede pretender que los alumnos entren en la cultura científica sin enseñarles a plantearse problemas, a hacerse preguntas, y sobre todo, a distinguir cuales son interesantes.

De esta manera en algunos casos el docente no enseña a sus estudiantes a preguntar, y por tal motivo estos no han aprendido, puede ocurrir que en las clases solo se realizan pregunta las cuales se limitan a responder y no le damos la oportunidad de pensar, por ejemplo ¿Qué entiendes por? ¿Qué es la célula. Si miramos en la tabla 1 los ejemplos de preguntas elaboradas por los estudiantes en la salida por las dependencias del colegio y por el jardín tiende a ser preguntas fácticas que solo se responden con un sí, no o simplemente fáciles de responder.

Por consiguiente, todo esto se presenta, porque en las clases poco se utiliza la formulación de preguntas y si se utiliza no despiertan el interés y la curiosidad de los alumnos; así como expresa Furman, M (2014) "la importancia de utilizar estrategias de enseñanza en las que las preguntas sean las principales puertas de entrada para el aprendizaje de las Ciencias Naturales", también dice que los docentes deben acercar a los estudiantes preguntas para pensar y no meramente fácticas, que se responden con un dato, preguntas que sean investigables, presentarle a los estudiantes preguntas que generen o representen desafíos, que representen casos y que contengan cuestiones intrigantes, que a los chicos les den ganas de responder.

De la misma manera los lineamientos curriculares expresan lo siguiente:

"Uno de los factores inmensamente limitantes de nuestro sistema educativo es precisamente el tiempo tan escaso que le dedicamos a las preguntas en el desarrollo de los temas de clase...y si se realizan....ninguna de estas preguntas son del tipo que construyen conocimiento"

Según los datos evidenciados en la gráfica 2, se encontró que solo dos estudiantes realizaron preguntas de tipo tres, estos estudiantes se caracterizan por su grado de madurez, son participativos, tienen respuestas muy concretas y estructuradas cundo se les pregunta en clase.

Se puede observar en la tabla 1 que un $20 \%$ de los estudiantes objetos de estudio en algunas preguntas presentan problemas de redacción, lo cual implica que la pregunta no fue comprendida por el docente o no se entendió lo que el estudiante quiso expresar. Además, se identificó que la mayoría de las preguntas están orientadas a la indagación y obtener datos o conceptos.

También se hace necesario enseñarles a construir preguntas de investigación, la cual deben ser factible para ser contestada en un tiempo determinado, sencillo, reflexivo, comprensible, contextualizado y acorde con la realidad donde se desarrolla el evento investigativo y la necesidad que tiene quien investiga. Cuando inicialmente se les pide a los estudiantes que realicen preguntas se sorprenden, debido a que generalmente es el maestro quien pregunta y ellos responden, estas actividades sirven para que los alumnos se sientan o jueguen el papel de investigador, de esta manera construyen y desarrollan procesos críticos y reflexivos al emplear las preguntas como: ¿qué ?, ¿cómo hacerlo?, 
Bio - grafía. Escritos sobre la Biología y su Enseñanza. ISSN 2027-1034

Edición Extraordinaria. p.p. 1314 - 1324

Memorias del IX Encuentro Nacional de Experiencias en Enseñanza de la Biología y la

Educación Ambiental. IV Congreso Nacional de Investigación en Enseñanza de la Biología.

¿cuándo? y ¿Por qué lo hacen?, ¿qué pasaría? los estudiantes pueden dar sus propios significado a la realidad donde están inmersos, descubrir, analizar, sintetizar, relacionar conceptos y hallazgos.

\section{CONCLUSIONES}

Con todo lo expuesto anteriormente se puede decir que con frecuencia el docente no sabe formular preguntas y si lo hace, éstas carecen de significado para el estudiante 0 solo se limita a dar respuestas fáciles de contestar.

El docente deberá implementar estrategias innovadoras para enseñar y aprender a investigar, y superar el dogma que indica, sólo es científico aquel que investiga hechos o situaciones que se pueden cuantificar y medir.

La enseñanza de la biología basada en la observación permite que los estudiantes puedan observar fenómenos y plantearse preguntas que le permitan ampliar su conocimiento, formular explicaciones basadas en las observaciones.

El fortalecimiento de las habilidades de pensamiento científico: observar y preguntar, permite que los estudiantes se acerquen a las primeras etapas del proceso científico,

Si un estudiante indaga, es capaz de construir su propio aprendizaje, y puede formular y responder preguntas que le permiten ampliar su conocimiento, su vocabulario y podrá aproximarse a las actividades propias de la ciencia.

Finalmente, podemos decir, que para desarrollar el pensamiento en los estudiantes y permitir que estos sean críticos, se hace necesario darle más sentido a lo que aprenden. De esta forma serán capaces de tomar sus propias decisiones. Cabe destacar que el docente juega un papel relevante en este proceso de aprendizaje mediante la debida reflexión de su quehacer pedagógico.

\section{REFERENTES}

Perkins, D ( ) Como hacer visible el pensamiento. (León, P \& Barrera, M., Trad.). Consultado en: 5 de mayo de 2017. Recuperado de:www.academia.edu/6680891/CÓMO_HACER_VISIBLE_EL_PENSAMIENTO.

Monsalve, J y Montoya, J (2008). Estrategias didácticas para fomentar el pensamiento crítico en el aula. Revista Virtual Universidad Católica del Norte. No.25. consultado el: 5 de mayo del 2017. Recuperado de: http://revistavirtual.ucn.edu.co/], ISSN 0124-582.

Santelices, L. (1989). Metodología de Ciencias Naturales para la Enseñanza Básica. Santiago de Chile: Andrés Bello.

Camacho, H. Casilla, D. \& Finol, M. (2008). La indagación: una estrategia innovadora para el aprendizaje de procesos de investigación. Laurus. Vol. 14, número 25. Consultado 
Bio - grafía. Escritos sobre la Biología y su Enseñanza. ISSN 2027-1034

Edición Extraordinaria. p.p. 1314 - 1324

Memorias del IX Encuentro Nacional de Experiencias en Enseñanza de la Biología y la Educación Ambiental. IV Congreso Nacional de Investigación en Enseñanza de la Biología.

en: 15 de septiembre de 2017. Recuperado de: www.redalyc.org/pdf/761/76111491014.pdf

Dewey, J. (1965). La Relación Teoría Práctica en Educación. Buenos Aires. Editorial Losada.

MEN. (1998). lineamientos curriculares: Ciencias Naturales y Educación Ambiental. Bogotá.

Furman, M., \& García, S. (2014). Categorización de preguntas formuladas antes y después de la enseñanza por indagación. Praxis y saber, 75-91.

Acevedo J.2004 Revista Eureka sobre Enseñanza y Divulgación de las Ciencias, vol. 1, núm. 1, enero, pp. 3-16.

Furió, C., Guisasola Aranzábal, J., Vilches Peña, A., \& Romo, V. (2001). Finalidades de la enseñanza de las ciencias en la secundaria obligatoria:¿ alfabetización científica o preparación propedéutica?. México:

Stenhouse. L(1920) La investigación del currículo. España: Ediciones Morata.S.A 